\title{
Preeclampsia, Placental Insufficiency, Autism Spectrum Disorder or Developmental Delay ... and Antiphospholipid Antibodies?
}

\author{
Cheryl K. Walker, MD ${ }^{1,2}$, Paul Ashwood, PhD ${ }^{2,4}$, and Irva Hertz-Picciotto, PhD $^{2,3}$ \\ ${ }^{1}$ Division of Maternal Fetal Medicine, Department of Obstetrics and Gynecology, School of \\ Medicine, University of California, Davis \\ ${ }^{2}$ MIND (Medical Investigation of Neurodevelopmental Disorders) Institute, University of California, \\ Davis \\ ${ }^{3}$ Department of Public Health Sciences, School of Medicine, University of California, Davis \\ ${ }^{4}$ Department of Medical Microbiology and Immunology, University of California, Davis
}

Dr. Carbillon has suggested that antiphospholipid antibodies (aPLs) in the mother and/or child might be confounding our recently published findings from the CHARGE case-control study that preeclampsia, particularly severe disease with placental insufficiency, was associated with autism spectrum disorder (ASD) and developmental delay (DD). ${ }^{1} \mathrm{We}$ hypothesized a role for suboptimal placentation to explain our findings. Indeed, poor placentation resulting from defective endovascular decidual trophoblastic invasion is a central mechanism in mothers with antiphospholipid syndrome (APS).

Individuals with APS have circulating aPLs (primarily lupus anticoagulant, anti-cardiolipin and anti- $\beta_{2}$-glycoprotein-I antibodies) which cause thrombosis and are often present in other autoimmune conditions. In pregnancy, APS is associated with recurrent pregnancy loss and fetal death, and gestations that do survive often are complicated by intrauterine growth restriction, preterm birth and preeclampsia.

In the European registry of women with APS, 4 of 134 children were diagnosed with neurobehavioral conditions, one each with autism, hyperactivity, feeding disorder with language delay, and axial hypotony with psychomotor delay. ${ }^{2}$ This series did not include mothers without APS and was insufficiently powered to address neurobehavioral impacts. Further, outcomes fell within established prevalence figures for the population studied. Evidence for detrimental effects of aPL on neurobehavioral development from this study was limited, as all 4 of the children with developmental issues were negative for aPLs at birth, though the child with language delay had transient anti- $\beta_{2}$-glycoprotein-I at 3-9 months and another with hyperactive behavior developed anti-cardiolipin at 24 months. The children with autism and language delay, however, were exposed to gestational diabetes, and the one with psychomotor delay was growth restricted. These findings support published mechanisms for fetal neurodevelopmental compromise, including inadequate placentation ${ }^{1}$

Corresponding Author: Cheryl K. Walker, MD, Associate Professor, Division of Maternal Fetal Medicine, Department of Obstetrics \& Gynecology, MIND Institute, University of California Davis School of Medicine, 4869 Y Street, Suite 2500, Sacramento, CA 95817, Phone: 916-734-6670, FAX: 916-734-6666, ckwalker@ucdavis.edu. 
and maternal autoimmune ${ }^{3}$ and/or metabolic dysfunction, ${ }^{4}$ leaving unanswered the question of whether fetal or childhood aPL exposure could have detrimental effects on neurobehavioral development.

Our group recently published on elevated aPL levels in 2-5 year olds with ASD enrolled in CHARGE compared to age-, sex- and geography-matched typically-developing children. ${ }^{5}$ Dr. Carbillon's question motivated us to investigate obstetric complications in association with the child's propensity to develop aPLs and subsequent neurodevelopmental diagnosis among 93 CHARGE Study children in whom antibodies were analyzed. Twenty-two percent experienced a clinical condition suggestive of placental insufficiency (preeclampsia, preterm birth or small-for-gestational-age birthweight). While these conditions were slightly more common in children with $\mathrm{DD}$, their prevalence did not differ by outcome ( $P=0.78)$.

Comparing aPL levels in children from pregnancies with and without placental insufficiency indicated a trend toward decreased anti-cardiolipin antibodies in the subset of children with ASD exposed to preeclampsia $(P=0.07)$, but no difference in anti- $\beta_{2}$-glycoprotein-I levels. Overall, there was no evidence linking clinical obstetric events accompanied by placental insufficiency to aPL levels in children with ASD, DD or TD in our small sample. We did not measure aPL in their mothers, and none of the women was diagnosed with APS.

The potential for aPL exposure, during fetal and early infancy periods from maternal transplacental transfer or in childhood through de-novo production, to alter neurodevelopment remains an intriguing hypothesis worthy of further scrutiny.

\section{References}

1. Walker CK, Krakowiak P, Baker A, Hansen RL, Ozonoff S, Hertz-Picciotto I. Preeclampsia, Placental Insufficiency, and Autism Spectrum Disorder or Developmental Delay. JAMA pediatrics. 2014 Dec 8.

2. Mekinian A, Lachassinne E, Nicaise-Roland P, et al. European registry of babies born to mothers with antiphospholipid syndrome. Annals of the rheumatic diseases. 2013 Feb; 72(2):217-222. [PubMed: 22589374]

3. Gesundheit B, Rosenzweig JP, Naor D, et al. Immunological and autoimmune considerations of Autism Spectrum Disorders. Journal of autoimmunity. 2013 Aug.44:1-7. [PubMed: 23867105]

4. Krakowiak P, Walker CK, Bremer AA, et al. Maternal metabolic conditions and risk for autism and other neurodevelopmental disorders. Pediatrics. 2012 May; 129(5):e1121-e1128. [PubMed: 22492772]

5. Careaga M, Hansen RL, Hertz-Piccotto I, Van de Water J, Ashwood P. Increased anti-phospholipid antibodies in autism spectrum disorders. Mediators of inflammation. 2013; 2013:935608. [PubMed: 24174712] 